\title{
Management of hereditary breast cancer: surgeon's perspective
}

\author{
Ava Kwong* \\ Chairman and Founder of The Hong Kong Hereditary Breast Cancer Family Registry \\ and Hong Kong Hereditary and High Risk Breast Cancer Programme, Assistant \\ Dean, Associate Professor and Chief of Breast Surgery, Department of Surgery, \\ The University of Hong Kong \\ Chief of Breast Surgery, Hong Kong University Shenzhen Hospital
}

DOI: http://dx.doi.org/10.19106/JMedScieSup004804201606

\section{ABSTRACT}

Mutations due to hereditary-related genes such as BRCA1, BRCA2, TP53 and PTEN confer greater risk of developing breast cancer. BRCA mutations were also found to be related to ovarian cancer. The risk assessment based on genetic testing allows options of surveillance and prevention in high-risk patients, as well as guiding physicians in selecting specific treatment, such as targeted therapies and platinum base chemotherapy. Once an individual has been found to carry the BRCA mutation, the choice of management might also vary. Moreover, the availability of genetic testing and other novel methods of testing, such as the Next Generation Sequencing techniques, have also provided options for clinicians. Breast Surgeons are most likely to be the first person encountering patients at their first presentation. It is important for breast surgeons to be actively involved in the referrals of high-risk patients for genetic testing, and subsequently in the planning of therapy, in a multidisciplinary setting. Basic principles of genetic testing and choice of management would be discussed in reference to the surgeon's perspectives.

Keywords: mutations, BRCA1. BRCA2. TP53, PTEN, NGS, multidisciplinary setting 